\title{
Automated Feature Analysis of Complex Geological Materials
}

\author{
Matt Hiscock $^{1 *}$, Simon Burgess ${ }^{1}, J$ hhn Zhang $^{1}$ and Alexandra Stavropoulou ${ }^{1,2}$ \\ 1. Oxford Instruments NanoAnalysis, High Wycombe, UK. \\ 2. Trinity College, Dublin, Ireland. \\ * Corresponding author: Matthew.Hiscock@oxinst.com
}

The decision on where on a sample an analysis should be performed is at the heart of all micro or nano analytical studies. In many cases this is a straightforward task which can simply be performed "by eye" on a secondary electron (SE) or backscattered electron (BSE) image on a scanning electron microscope (SEM). Whilst it is fine to use the incredible image processing capability of the human eye when working manually, this becomes impractical when the process of identifying where to perform an analysis must be automated. Instead, we must rely on computational algorithms to do the job for us.

In most cases it is possible to identify the specific features of interest by means of grey level thresholding of electron images. BSE images are most frequently used for this purpose as contrast in this imaging modality is related to average atomic number differences - meaning that compositional variations can be viewed. This makes it possible to identify multiple grains within particles as is common in geological analyses. There are however many cases where this thresholding becomes difficult if not impossible for a number of reasons. These reasons, in a geological context, include very small density differences between mineral phases, the presence of strong intergranular textures (e.g. cleavage) which may be identified as features owing to their different grey level or scratches or other sample damage caused during the sample preparation process. An example of a low density difference is shown in Figure 1.

Here, we consider a particular example of this - the exsolution textures in pyroxene in a sample from the Bushveld Igneous Complex (BIC) in South Africa. This rock has previously been characterised as an orthopyroxenite by hand specimen observations and cross polarized light microscopy. The sample was prepared by sectioning a representative portion of the hand specimen and polishing until it reached a thickness of $30 \mu \mathrm{m}$. The mineralogy determined by light optical microscopy (LOM) was found to be OPX containing blebs of CPX, interstitial plagioclase, sulphide minerals and chromite. In addition, various other sulphide phases have been identified with SEM equipped with energy dispersive x-ray spectroscopy (EDS). It has been observed that apparent pyroxene exsolution textures exist within the rock, indicative of the sample's cooling history.

Here, the densities, and so the appearance in a BSE image of the 2 phases making up the exsolution texture are very similar making identification and measurement of the proportions of the phases very difficult based on BSE imaging alone. Furthermore, the sample exhibits numerous cracks and scratches which can interrupt the measurement of grains in an imaging-based grain detection approach.

In order to overcome this problem, we have used a novel, data efficient mapping-based approach for the identification of mineral phases based on their composition instead of how they appear in electron images. In this approach, EDS maps are automatically acquired from either entire fields or specific regions of interest. This map data is then segmented by a fully automated algorithm to find areas of unique composition. Processing of a large area map across multiple fields is performed sequentially for 
each individual field and results are updated for every new field processed. This eliminates the need to "montage" the many fields into a potentially very large map for lengthy processing.

With this approach the isolation of small particles with unique chemical compositions from a large sample is a straightforward and fast process. This makes it possible to differentiate unique chemical distributions across the sample, and to provide a full table of statistics, detailing particle size, shape, and chemistry. The result of this process can be clearly seen in Figure 2.

By utilising this approach, otherwise indistinguishable phases have been identified and analysed automatically using a feature analysis approach, providing valuable measurements of the relative proportions of the phases (Table 1). It is applicable to both solid sections and particulated samples and provides an advanced survey tool for feature detection when it would otherwise be difficult or impossible.

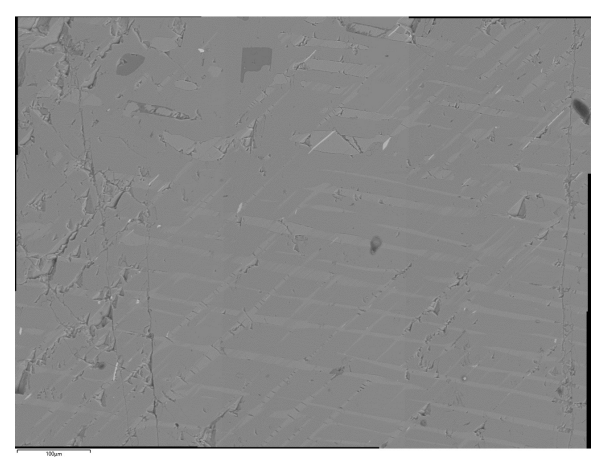

Figure 1. Low grey level constrast between phases makes accurate detection difficult, if not impossible

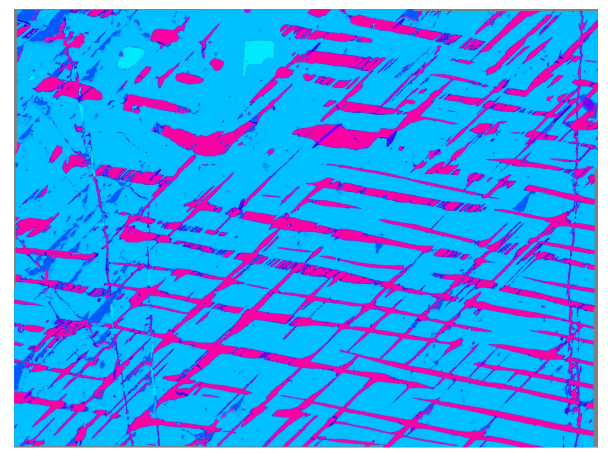

Figure 2. Identification of the exsolved phases based on composition - no imaging data was used in the detection of these phases.

\begin{tabular}{|l|r|rr|}
\hline Class & $\begin{array}{c}\text { Class Features } \\
\text { Area }(\mathbf{s q} . \boldsymbol{\mu m})\end{array}$ & $\begin{array}{r}\text { \% } \\
\text { Area }\end{array}$ & Features \\
\hline OPX & $8.05 \mathrm{E}+04$ & & 18.14 \\
\hline CPX & $3.28 \mathrm{E}+05$ & 74.06 \\
\hline Feldspar & $1.83 \mathrm{E}+03$ & 0.41 \\
\hline Unequilibrated & $8.87 \mathrm{E}+03$ & 2 \\
\hline
\end{tabular}

Table 1. Summary of the relative proportions of the identified phases 